\title{
The Coexistence of Second and Third Malignancies in Adult-Onset Cancer Patients
}

\author{
Jacopo Giuliani
}

Published online: 1 February 2014

(C) Springer Science+Business Media New York 2014

The coexistence of second and third malignancies is a question poorly addressed by scientific literature and medical debates. The frequency of this association and the spectrum of neoplasms involved have not been sufficiently analyzed; in fact, most of publications describe single case report [1] and rarely case series $[2,3]$. Below, it is reported as the following experience, derived from retrospective analysis of patients who died in 2011 (from January to December), residents in our Local Unit Social Health (catchment area of approximately 150,000 inhabitants), diagnosed with cancer as a cause of death. No results like the above have been published until now. Among 1,496 patients who died in the reference period, 455 patients $(30.4 \%)$ have been diagnosed with cancer as cause of death. From these, median age at the time of death was 77.6 years (range 2-97); 280 patients (61.5\%) were male and 175 patients $(38.5 \%)$ were female. Seven patients $(1.5 \%$ of patients diagnosed with cancer) had a diagnosis of second (4 patients) or third ( 3 patients) malignancies; median age at the time of death was 74.0 years (range 56-85). Among these (patients with second or third malignancies), 4 patients (57.1\%) had a colon cancer. These data imply that the given issue was not reported in literature, which may have reflected not only in terms of speculative discussion but also in terms of prognosis. The patterns of second primary malignancies in survivors of adult-onset cancer differ in terms of the observed types of second tumors, magnitude of risks, latency periods, and influence of modifying factors. Possible explanations for these variations include the genetic underpinnings and etiology of the first cancer diagnosis, variations in treatment approaches, and contributions and modifying effects of exposures to environmental factors (e.g., tobacco or alcohol) [4].

J. Giuliani $(\bowtie)$

Department of Oncology, Mater Salutis Hospital, ASL 21 della

Regione Veneto Via Gianella 1, 37045 Legnago, VR, Italy

e-mail: giuliani.jacopo@alice.it
Further studies are required to clarify the molecular and genetic mechanisms of carcinogenesis and progression associating with secondary and third malignancies, especially concerning gastrointestinal cancer [5]. We also foresee that some characteristics can be further investigated in order to define a possible predictive model of association between different malignancies, particularly if we consider as nonrandom causal association the coexistence as a possible oncologic model for the recognition of a syndromic setting and synchronous occurrence with certain tumors. Although differences exist in the risk of second malignancies across the age spectrum, lessons learned from one age group may potentially be applicable to other age groups as treatments evolve, followup time increases, and our understanding of secondary carcinogenesis improves [4]. Because of the limited number of cases, we cannot exclude an incidental relationship, but this association should be considered. Larger studies are needed to confirm such an interesting observation.

Conflict of Interest None declared.

\section{References}

1. Jimenez VH. Coexistence between renal cell cancer and Hodgkin's lymphoma: a rare coincidence. BMC Urol. 2006;20:10-4.

2. Ustun C, Reid-Nicholson M, Nayak-Kapoor A, et al. Plasmablastic lymphoma: CNS involvement, coexistence of other malignancies, possible viral etiology, and dismal outcome. Ann Hematol. 2009;88:351-8.

3. Laurenti L, Tarnani M, Nichele I, et al. The coexistence of chronic lymphocytic leukemia and myeloproliperative neoplasms: a retrospective multicentric GIMEMA experience. Am J Hematol. 2011;86: 1007-12.

4. Ng AK, Kenney LB, Travis LB. Secondary malignancies across the age spectrum. Semin Radiat Oncol. 2010;20:67-78.

5. Giuliani J, Marzola M, Indelli M, et al. Gastrointestinal stromal tumors and other malignancies: a case series. J Gastrointest Cancer. 2012;43:634-7. 